\title{
Predictive control for reliable microgrid energy management under uncertainties
}

\author{
I. Prodan \\ Chair on Systems Science and the Energetic Challenge, European Foundation for New Energy, EDF, \\ Ecole Centrale Paris, Grande Voie des Vignes and Supélec, Gif sur Yvette, France
}

\section{E. Zio}

Chair on Systems Science and the Energetic Challenge, European Foundation for New Energy, EDF, Ecole Centrale Paris, Grande Voie des Vignes and Supélec, Gif sur Yvette, France

Department of Energy, Politecnico di Milano, Italy

\begin{abstract}
This paper proposes the basic elements of an approach to reliable microgrid energy management based on receding horizon control. The microgrid considered for exemplification is connected to an external grid via a transformer and contains a local consumer, a renewable generator (wind turbine) and a storage facility (battery). In order to minimize costs, a scheduling of the battery usage needs to be determined. To this end, a predictive control framework is proposed here, which allows to naturally take into consideration cost values, power consumption and generation profiles, as well as functioning constraints under uncertainty due to variations in the environment (wind speed), to failures and subsequent repairs of the generator. The proposed approach is validated through simulation results using real numerical data for a reliability test system.
\end{abstract}

\section{INTRODUCTION}

Renewable and sustainable energy management represents an active area of research nowadays (Alanne \& Saari 2006), (Zio \& Aven 2011), (Lund 2007). All traditional energy supplies of Earth are finite and suffer from a "diminishing returns" curse. In contrast, solar and wind energy are practically limitless but require engineering solutions for a sustainable development.

Microgrids can be key solutions for integrating renewable and distributed energy resources, as well as distributed energy-storage systems (Chen, Duan, Cai, Liu, \& Hu 2011), (Katiraei \& Iravani 2006).

The present paper is focused on microgrid energy dynamical systems. For these systems, it is no longer possible to control each subsystem without taking in consideration its interactions with the other subsystems (Negenborn, De Schutter, \& Hellendoorn 2006). The relations between the subsystems can be linked to their dynamical interactions (Logenthiran, Srinivasan, \& Wong 2008), (Colson, Nehrir, \& Gunderson 2010). In the control framework for microgrid energy systems it is necessary to take into account not only exogenous factors (e.g., change in consumer load, wind speed, price profile, etc.) but also the internal (state) dynamics and the structural properties of the individual components (as wind or solar energy equipment, on-site storage etc.), which may change (stochastically) due to degradation, failure, aging and so on.

Various approaches for the energy management within a microgrid are reported in the literature. For example, (Jimeno, Anduaga, Oyarzabal, \& de Muro 2011), (Kuznetsova, Culver, \& Zio 2011), (Krause, Beck, Cherkaoui, Germond, Andersson, \& Ernst 2006), (Weidlich \& Veit 2008) propose an agentbased modeling approach to model microgrids and to analyze by simulation the interactions between individual intelligent decision-makers. Energy management of hybrid renewable energy generation was proposed in (Colson, Nehrir, \& Gunderson 2010), (Jun, Junfeng, Jie, \& Ngan 2011). In thesepapers, the long-term goals are focused on the efficient use of electricity within microgrids, e.g., the planning of battery scheduling to store the electricity locally generated from renewable sources and reuse it during periods of high electricity demand. It is important to mention that in these works the decision framework was developed under deterministic conditions, e.g., those of a typical day in summer, and without taking into account explicitly the dynamics of the individual components of the microgrids. 
A widely used technique in the control community to manage the dynamics of systems affected by uncertainties in the behavior of their components is Model Predictive Control (MPC) (see, for instance, (Rawlings \& Mayne 2011), (Richalet \& O'Donovan 2009) for basic notions about MPC), due to its ability to handle control and state constraints while offering good performance specifications. Typically, in MPC, the objective (or cost) function penalizes deviations of the states and inputs from their reference values, while the constraints are enforced explicitly (Goodwin, Seron, \& De Dona 2005). Recently, MPC is being considered for refrigeration systems (Hovgaard, Larsen, \& Jorgensen 2011), for power production plants (Halvgaard, Poulsen, Madsen, \& Jorgensen 2012), (Edlund, Bendtsen, \& Jørgensen 2011) and transportation networks (Negenborn, De Schutter, \& Hellendoorn 2008).

In the present paper, we address the problem of reliable energy management of a microgrid by proposing a predictive control framework. To the best of the authors' knowledge, there does not exist a similar method in the open literature. The microgrid considered as example is connected to an external grid via a transformer and contains a local consumer, a renewable generator (wind turbine) and a storage facility (battery). The underlying management setting is one of multi-criteria decision-making regarding battery scheduling with the objectives of increasing utilization rate of the battery during high electricity demand (i.e., decrease of the electricity purchase from the external grid) and increasing the utilization rate of the wind turbine for local use (i.e., increase of the consumer independence from the external grid). The proposed predictive control framework takes into account the uncertain scenarios under which the microgrid operates, that is, it accounts for the stochasticity in the load, in the environmental conditions affecting the available wind power output and in the operational conditions of the wind power generator, due to failures and repairs.

First the consumers' load, the wind generator and the price profiles are provided. Then, a constrained multi-objective optimization problem is developed and solved in order to follow the predicted profiles for a reliable energy management.

\subsection{Notation}

The following notations will be used throughout the paper. Denote $\mathbb{B}_{p}^{n}=\left\{x \in \mathbb{R}^{n}:\|x\|_{p} \leq 1\right\}$ as the unit ball of norm $p$, where $\|x\|_{p}$ is the $p$-norm of vector $x$. Minkowski's addition of two sets $\mathcal{X}$ and $\mathcal{Y}$ is defined as $\mathcal{X} \oplus \mathcal{Y}=\{x+y: x \in \mathcal{X}, y \in \mathcal{Y}\}$. Let $x(k+1 \mid k)$ denote the value of $x$ at time instant $k+1$, predicted upon the information available at

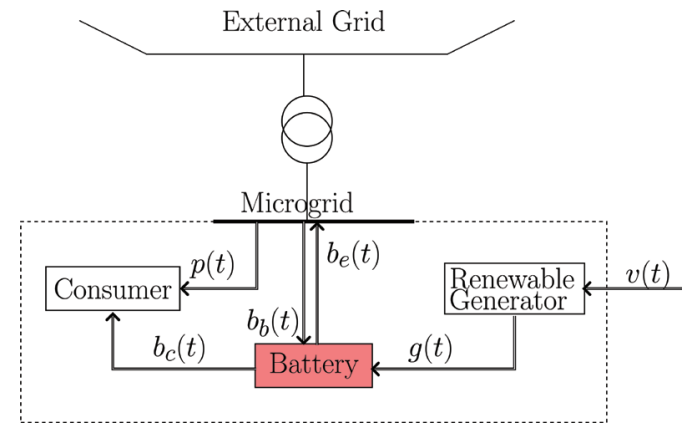

Figure 1. Microgrid architecture.

time $k \in \mathbb{N}$. We write $R \succ(\succeq) 0$ to denote that $R$ is a positive (semi)definite matrix. Let $s_{\max }$ denote the maximum number of steps for which an optimization problem is solved.

\section{PRELIMINARIES}

Consider a microgrid as in Figure 1, which includes a local consumer (e.g., large cooling houses), a renewable generator (e.g., wind turbine) and a storage facility (e.g., battery). The microgrid is connected to the external grid via a transformer. The goal is to plan the battery schedule in order to achieve the consumer objectives:

- increase the utilization rate of the battery during high electricity demand (i.e., decrease the electricity purchase from the external grid).

- increase the utilization rate of the generator for local use (i.e., increase the consumer independence from the external grid).

The interactions between the independent components of the microgrid are one of the most important factors in accomplishing the consumer objectives. Let us provide a brief description of them:

$-g(t) \in \mathbb{R}[\mathrm{W}]$ represents the electricity power transmitted by the renewable generator to the battery at time step $t$.

$-b_{c}(t) \in \mathbb{R}[\mathrm{W}]$ represents the electricity power transmitted by the battery to the consumer at time step $t$. In order to maximize the utilization of the battery within the microgrid the consumer takes electricity from the local renewable generator through the battery. Next, the battery can be charged from the external grid or can also give electricity to the external grid.

- $b_{e}(t) \in \mathbb{R}[\mathrm{W}]$ represents the electricity power transmitted by the battery to the external grid at time step $t$. It is possible to sell electricity to the external grid when the level of charge 
in the battery is deemed sufficient for covering local needs.

- $b_{b}(t) \in \mathbb{R}[\mathrm{W}]$ represents the electricity power transmitted by the external grid to the battery at time step $t$.

- $p(t) \in \mathbb{R}[\mathrm{W}]$ represents the electricity power transmitted by the external grid to the consumer at time $t$. Here, the transformer provides electricity power from the external grid as well as information about the electricity market price, which plays an important role as the consumer can decide to take energy when the price is low. Therefore, the consumer has also the possibility to take electricity from the external grid when the renewable resource is not available (or sufficient).

Finally, the ultimate goal is to control the battery such that all the consumers objectives are fulfilled while considering all the interactions between the independent components of the electrical grid.

\subsection{Dynamic models of the microgrid components}

The controllable component here is the battery. For describing its dynamics we can start first by considering a straightforward description:

$b(t+1)=b(t)+\left[b_{b}(t)+g(t)-b_{c}(t)-b_{e}(t)\right] \Delta t$,

where $b(t) \in \mathbb{R}[W h]$ represents the level of battery charge at time step $t$ and $b_{b}(t) \in \mathbb{R}[\mathrm{W}], g(t) \in \mathbb{R}[\mathrm{W}]$, $b_{c}(t) \in \mathbb{R}[\mathrm{W}], b_{e}(t) \in \mathbb{R}[\mathrm{W}]$, the electricity power quantities previously described (see also Fig. 1).

Consider also the generator system, whose dynamics can be described as:

$g(t+1)=f(g(t), v(t))$,

where $f(\cdot, \cdot): \mathbb{R} \rightarrow \mathbb{R}$ is the dynamical system, $g(t) \in \mathbb{R}$ the electricity power given by the generator and $v(t) \in \mathbb{R}$ the wind speed. Note that $f(\cdot, \cdot)$ is nonlinear, that is, the output of the generator depends nonlinearly on the wind speed and can be roughly partitioned into three regimes of functioning (starting-up, nominal functioning and dangerous wind level), as shown in Figure 2.

\subsection{Constraints description}

From Figure 1 the consumer can take electricity from two sources, i.e., the battery and the external grid. Therefore, for a reliable (adequate) energy system it is necessary to ensure that at time $t$ the electricity purchased from these twosources satisfies the following condition:

$b_{c}(t)+p(t) \geq d(t)$.

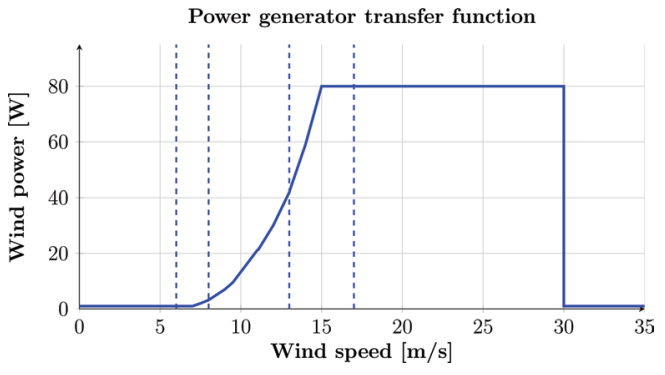

Figure 2. Illsutration of the reference power curve for the wind turbine depending on the wind speed.

The energy stored in the battery at time $t$ needs to remain between some bounds; consequently, the following condition is imposed:

$B_{\min } \leq b(t) \leq B_{\max }$,

where $B_{\min }, B_{\max } \in \mathbb{R}$

Furthermore, the rate of battery charge at time $t$ needs to remain between some bounds as described by the following condition:

$D_{\min } \leq \Delta b(t) \leq D_{\max }$,

where $D_{\text {min }}, D_{\max } \in \mathbb{R}$

\subsection{Cost function description}

Consider $e(t) \in \mathbb{R}[\$ / W h]$ the electricity market price at time step $t$. Then, the total energy cost at time $t$ is:

$C(t)=e(t) \cdot \Delta t\left[b_{b}(t)+p(t)-b_{e}(t)\right]$,

where a small value of $C(t)$ means small energy drawn from the external grid.

\subsection{Profile construction}

All the elements of interest are characterized by certain profiles of reference.

- $d(t)$, consumer load profile

Arguably, the most important is the profile characterizing the consumer load. Taking into account the weekly, daily and hourly variability it is possible to predict a reference load. Therefore, in order to predict the reference load of the consumer we considered a top-down approach based on available statistical collections of electricity consumption (the real numerical data of a reliability test system task force are found in (Grigg, Wong, Albrecht, Allan, 
Bhavaraju, Billinton, Chen, Fong, Haddad, \& Kuruganty 1999)). Considering $r_{w}^{\text {peak }}(\%)$ the weakly peak of power demand in percent of annual peak, $r_{d}^{\text {peak }}(\%)$ the daily peak of power demand in percent of weekly peak, $r_{h}^{\text {peak }}(\%)$ the hourly peak of power demand in percent of daily peak and $d^{\text {peak }}[\mathrm{W}]$ the total electricity power demand over a year, then the consumer load satisfies the following relation:

$$
d(t)=\frac{r_{h}^{\text {peak }}(l)}{\sum_{l=1}^{24} r_{h}^{\text {peak }}(l)} \cdot \frac{r_{d}^{\text {peak }}(j)}{\sum_{j=1}^{7} r_{d}^{\text {peak }}(j)} \cdot \frac{r_{w}^{\text {peak }}(i)}{\sum_{i=1}^{52} r_{w}^{\text {peak }}(i)} \cdot d^{\text {peak }} \cdot \Delta t
$$

with $l=1: 24$, the hours indices in a day, $j=1: 7$, the days indices in a week and $i=1: 52$, the week indices in a year ${ }^{1}$.

Figure 3 depicts the reference load of the consumer as described in (7).

\section{$-v(t)$, wind speed profile}

Furthermore, we also need to specify a profile for the generator power output. Note that this output depends directly on the wind profile, which has to be estimated from meteorological data and information. Figure 4 depicts the considered wind speed profile, the numerical data taken from (Grigg, Wong, Albrecht, Allan, Bhavaraju, Billinton, Chen, Fong, Haddad, \& Kuruganty 1999).

Note that the wind speed correlates with a nonlinear profile for the generator output. As seen in Figure 2, we can divide in different areas of functioning: low-speed where the generator is in the process of starting-up, nominal functioning and extreme conditions where the generator is decoupled.

\section{- $g(t)$, power generator profile}

We consider the power generator given by the following power curve transformation (Justus, Hargraves, \& Yalcin 1976):

$$
g(t)= \begin{cases}0, & \text { if } v<v_{c i}, \\ P_{r} \cdot \frac{v(t)-v_{c i}}{v_{r}-v_{c i}} \cdot \Delta t, & \text { if } v_{c i} \leq v(t)<v_{r}, \\ P_{r} \cdot \Delta t, & \text { if } v_{r} \leq v<v_{c o}, \\ 0, & \text { if } v>v_{c o},\end{cases}
$$

where $v(t)[\mathrm{m} / \mathrm{s}]$ is the working wind speed at time step $t$ of 1 hour, $v_{c i}[\mathrm{~m} / \mathrm{s}], v_{r}[\mathrm{~m} / \mathrm{s}]$ and $v_{c o}[\mathrm{~m} / \mathrm{s}]$ are the cut-in, rated and cut-off wind speeds, respectively, and $P_{r}[W]$ is the rated power of the wind turbine.

\footnotetext{
${ }^{1}$ Note that the consumer load profile takes into account seasonal numerical data, thus it can be used to adapt to any system peaking season one desires to model.
}

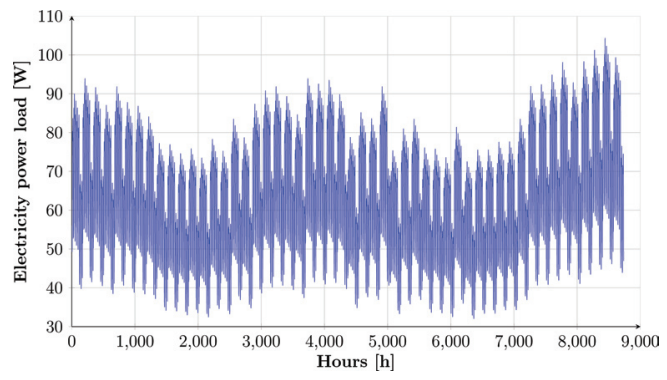

Figure 3. Consumer load profile.

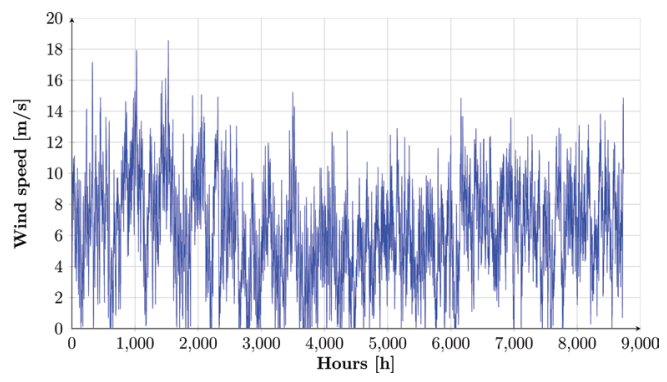

Figure 4. Wind speed profile.

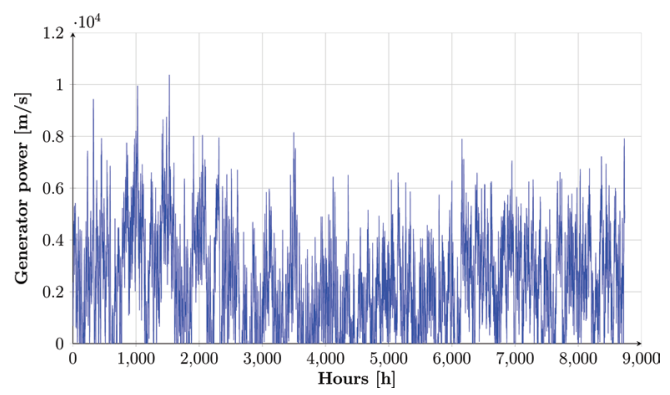

Figure 5. Power generator profile.

Figure 5 depicts the power generator profile with the numerical data $P_{r}=6000 \mathrm{~W}, v_{c i}=3 \mathrm{~m} / \mathrm{s}$, $v_{r}=12 \mathrm{~m} / \mathrm{s}, v_{c o}=20 \mathrm{~m} / \mathrm{s}$.

- $e(t)$, electricity market price profile

Lastly, we need to provide the evolution of the prices on the electricity market. Again we use existing data about the previous movements of the market prices (Grigg, Wong, Albrecht, Allan, Bhavaraju, Billinton, Chen, Fong, Haddad, \& Kuruganty 1999): Figure 6 depicts the evolution of the price on the electricity market in a day. 


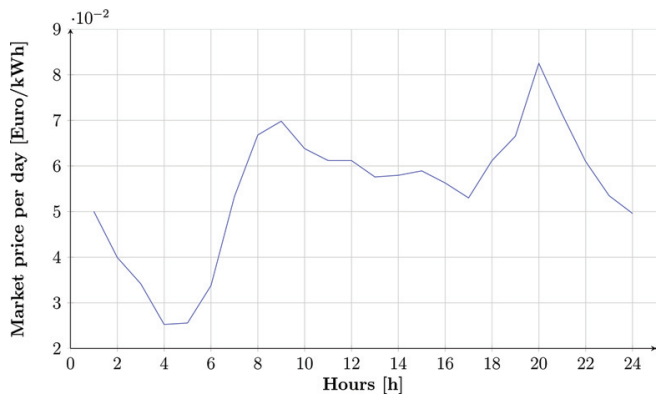

Figure 6. Electricity market price profile.

\section{OPTIMIZATION-BASED CONTROL MECHANISM FOR BATTERY SCHEDULING}

For a reliable energy management of the microgrid by battery scheduling, we consider the recursive construction of an optimal open-loop control sequence $\mathbf{u}=\left\{u(k), u(k+1), \ldots, u\left(k+N_{\mathrm{p}}-1\right)\right\}$, with $u(k)=\left[p^{T}(k) b_{e}^{T}(k) b_{c}^{T}(k) b_{b}^{T}(k)\right]^{T}$ over a finite constrained receding horizon, which leads to a feedback control policy by the effective application of the first control action as system input:

$u^{*}=\arg \min _{[u(k)]_{t=k: k+N_{p}}} \sum_{t=k}^{k+N_{\mathrm{p}}} C(t)$,

subject to the set of constraints:

$$
\begin{aligned}
& b(t+1)=b(t)+\left[b_{b}(t)+g(t)-b_{c}(t)-b_{e}(t)\right] \Delta t, \\
& g(t+1)=f(g(t), v(t)), t=k: k+N_{p}, \\
& b_{c}(t)+p(t) \geq d(t), t=k: k+N_{p}, \\
& B_{\text {min }} \leq b(t) \leq B_{\text {max }}, t=k: k+N_{p}, \\
& D_{\text {min }} \leq \Delta b(t) \leq D_{\text {max }}, t=k: k+N_{p}, \\
& C(t)=e(t) \cdot \Delta t\left[b_{b}(t)+p(t)-b_{e}(t)\right], t=k: k+N_{p} .
\end{aligned}
$$

Note that the price $e(t)$ is variable, therefore, in (9) we have a variable cost function.

\section{SIMULATION RESULTS}

We consider the dynamic model (1) for the battery, the discretization step $\Delta t=1$ hour and the profiles described in Section 2.4. The battery charge bounds are $B_{\min }=0 \mathrm{kWh}, B_{\max }=100 \mathrm{kWh}$ and the battery charge variation bounds $D_{\text {min }}=-5 \mathrm{kWh}$, $D_{\text {max }}=5 \mathrm{kWh}$. Also, the control signals $b_{b}(t), b_{c}(t)$, $b_{e}(t), p(t)$ need to stay between some bounds, i.e., $[0,50] \mathrm{kWh}$.
The figures of this section illustrate several of the signals of interest. First, Figure (7) shows the level of battery charge.

Next, Figure 8 (top right) shows the consumer power demand (in blue) $d(t)$, power given by the battery to the consumer (in green) $b_{c}(t)$, the power given by the external grid to the consumer (in red) $p(t)$. Notice that the sum of the last two (i.e., $\left.b_{c}(t)+p(t)\right)$ is depicted in dashed blue and is always equal with the consumer power demand which means that the consumer is never left waiting for electricity power.

The same Figure 8 (bottom left) depicts both, the power given by the battery to the external grid (in green) $b_{e}(t)$ and the power given by the external grid to the battery (in red) $b_{b}(t)$. Also in blue we show how much power is drawn overall from the grid $p(t)+b_{b}(t)-b_{e}(t)$ (power given from the external grid to the consumer + power given from the grid to the battery-power given from the battery to the grid).

Finally, Figure 8 (bottom right) shows the value of the cost at each time instant.
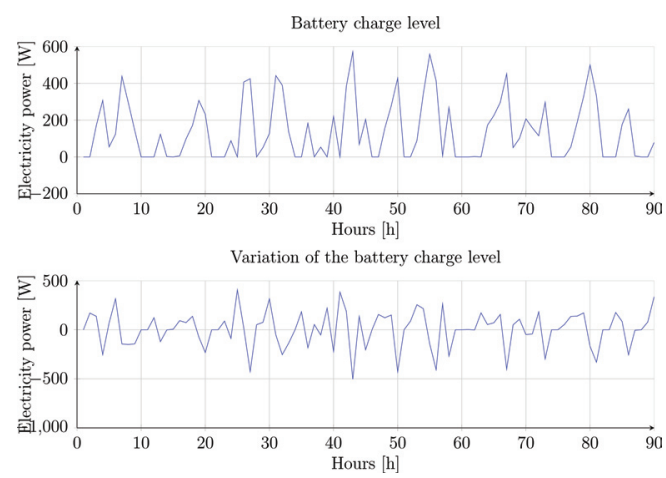

Figure 7. Battery charge level and its variation.

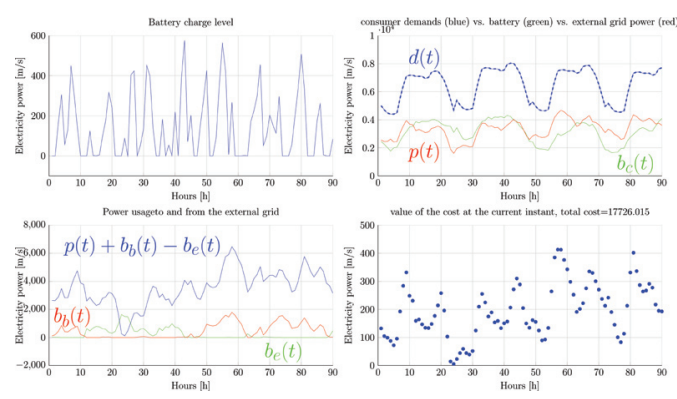

Figure 8. Powe usage. 


\section{FUTURE MODEL DEVELOPMENTS}

The development of the model must be extended to include some other objectifs. More precisely, the battery can loose its capacity in time, as for example it charges more rapidly when it is more empty and more slowly when it is more loaded. Also, the loading process may take a certain finite amount of time. Moreover, a battery does not remain charged for all the time, therefore there is the possibility for charge leakage.

Furthermore, the profiles can be more complicated. For example, there exists the possibility to have different electricity market prices for selling and buying. Also, a more precise definition of power production $g(t)$ needs to be developed, accounting for components faults and uncertainties.

Finally, in the predictive control context, we need to take into account realistic values for $d(t)$, $e(t), v(t)$. We need to introduce disturbances, therefore a tube MPC approach can be employed (Mayne, Kerrigan, \& Falugi).

\section{REFERENCES}

Alanne, K. \& A. Saari (2006). Distributed energy generation and sustainable development. Renewable and Sustainable Energy Reviews 10 (6), 539-558.

Chen, C., S. Duan, T. Cai, B. Liu, \& G. Hu (2011). Smart energy management system for optimal microgrid economic operation. IET renewable power generation 5 (3), 258-267.

Colson, C., M. Nehrir, \& R. Gunderson (2010). Multiagent microgrid power management. In Proceedings of the 18th IFAC World Congress, pp. 77-82.

Edlund, K., J. Bendtsen, \& J. Jørgensen (2011). Hierarchical model-based predictive control of a power plant portfolio. Control Engineering Practice 19 (10), $1126-1136$

Goodwin, G., M. Seron, \& J. De Dona (2005). Constrained control and estimation: an optimisation approach. Springer Verlag.

Grigg, C., P. Wong, P. Albrecht, R. Allan, M. Bhavaraju, R. Billinton, Q. Chen, C. Fong, S. Haddad, \& S. Kuruganty (1999). The ieee reliability test system-1996. a report prepared by the reliability test system task force of the application of probability methods subcommittee. Power Systems, IEEE Transactions on 14 (3), 1010-1020.

Halvgaard, R., N.K. Poulsen, H. Madsen, \& J. Jorgensen (2012). Economic model predictive control for building climate control in a smart grid. In IEEE PES Innovative Smart Grid Technologies (ISGT), pp. 1-6. IEEE.

Hovgaard, T., L. Larsen, \& J. Jorgensen (2011). Robust economic mpc for a power management scenario with uncertainties. In Proceedings of the 50th IEEE Conference on Decision and Control and European Control Conference (CDC-ECC), pp. 1515-1520. IEEE.
Jimeno, J., J. Anduaga, J. Oyarzabal, \& A. de Muro (2011). Architecture of a microgrid energy management system. European Transactions on Electrical Power 21 (2), 1142-1158.

Jun, Z., L. Junfeng, W. Jie, \& H. Ngan (2011). A multiagent solution to energy management in hybrid renewable energy generation system. Renewable Energy 36 (5), 1352-1363.

Justus, C., W. Hargraves, \& A. Yalcin (1976). Nationwide assessment of potential output from wind-powered generators. Journal of Applied Meteorology 15 (7), 673-678.

Katiraei, F. \& M. Iravani (2006). Power management strategies for a microgrid with multiple distributed generation units. IEEE Transactions on Power Systems 21 (4), 1821-1831.

Krause, T., E. Beck, R. Cherkaoui, A. Germond, G. Andersson, \& D. Ernst (2006). A comparison of nash equilibria analysis and agent-based modelling for power markets. International Journal of Electrical Power \& Energy Systems 28 (9), 599-607.

Kuznetsova, E., K. Culver, \& E. Zio (2011). Complexity and vulnerability of smartgrid systems. In Proceedings of the European Safety and Reliability Conference, pp. 2474-2482.

Logenthiran, T., D. Srinivasan, \& D. Wong (2008). Multi-agent coordination for der in microgrid. In Proceedings of the IEEE International Conference on Sustainable Energy Technologies, pp. 77-82. IEEE.

Lund, H. (2007). Renewable energy strategies for sustainable development. Energy 32 (6), 912-919.

Mayne, D., E. Kerrigan, \& P. Falugi. Robust model predictive control: advantages and disadvantages of tube-based methods. pp. 148-153.

Negenborn, R., B. De Schutter, \& H. Hellendoorn (2006). Multi-agent model predictive control of transportation networks. In Proceedings of the IEEE International Conference on Networking, Sensing and Control, pp. 296-301. IEEE.

Negenborn, R., B. De Schutter, \& J. Hellendoorn (2008). Multi-agent model predictive control for transportation networks: Serial versus parallel schemes. Engineering Applications of Artificial Intelligence 21 (3), 353-366.

Rawlings, J. \& D. Mayne (2011). Postface to model predictive control: Theory and design.

Richalet, J. \& D. O'Donovan (2009). Predictive Functional Control: Principles and Industrial Applications. Springer.

Weidlich, A. \& D. Veit (2008). A critical survey of agentbased wholesale electricity market models. Energy Economics 30 (4), 1728-1759.

Zio, E. \& T. Aven (2011). Uncertainties in smart grids behavior and modeling: What are the risks and vulnerabilities? how to analyze them? Energy Policy 39 (10), 6308-6320. 